\title{
A Locally Conservative Energy-Momentum Tensor in the General Relativity Based on a Cosmological Model without Singularity
}

\author{
Shihao Chen \\ Center for Quantum Sciences and School of Physics, Northeast Normal University, Changchun, China \\ Email: shchen@nenu.edu.cn
}

Received 24 December 2015; accepted 2 February 2016; published 5 February 2016

Copyright (C) 2016 by author and Scientific Research Publishing Inc.

This work is licensed under the Creative Commons Attribution International License (CC BY).

http://creativecommons.org/licenses/by/4.0/

c) (i) Open Access

\section{Abstract}

According to the conventional theory it is difficult to define the energy-momentum tensor which is locally conservative. The energy-momentum tensor of the gravitational field is defined. Based on a cosmological model without singularity, the total energy-momentum tensor is defined which is locally conservative in the general relativity. The tensor of the gravitational mass is different from the energy-momentum tensor, and it satisfies the gravitational field equation and its covariant derivative is zero.

\section{Keywords}

Energy-Momentum Tensor of Gravitational Field, Locally Conservative Energy-Momentum Tensor in General Relativity, Tensor of the Gravitational Mass, Quasi-Local Energy-Momentum Tensor

\section{Introduction}

The definition and local conservation of energy-momentum in the general relativity are two important and unsatisfactorily solved issues. Such an energy-momentum tensor which satisfies $\partial\left(\sqrt{-g} T^{\mu v}\right) \partial x^{v}=0$ has not been found up to now [1]-[5]. Thus, some physicists give up on the definition of the locally conservative energymomentum tensor and attempt to find a quasi-local energy-momentum tensor based on the principle of equivalence [6]-[8]. On the other hand, the cosmological singularity is hardly accepted, and the cosmological constant issue is not satisfactorily solved as well. In order to solve the two issues, a cosmological model without singularity had been proposed in Ref. [9]. Based on the model, we consider defining an energy-momentum tensor which satisfies $\partial\left(\sqrt{-g} T^{\mu v}\right) \partial x^{v}=0$ in the present paper.

According to the cosmological model, there are two sorts of matter which are called solid-matter (s-matter) and 
void-matter ( $v$-matter), respectively. Both are symmetric and the symmetric gauge group is $S U_{S}(5) \times S U_{V}(5)$ before the symmetry breaking. Both masses and energies are positive, but their contributions to the Einstein tensor $G_{\mu v}=R_{\mu \nu}-g_{\mu v} R / 2$ are opposite from each other. There is no other interaction between both except the interaction of Higgs fields when temperature is high enough and repulsion from each other. There are two sorts of breaking, i.e. $S$-breaking and $V$-breaking. But only one of the $S$-breaking and the $V$-breaking can occur in fact. For example, the $S$-breaking occurs. When the $S$-breaking occurs, elementary s-particles get their masses and form the given world. All $v$-particles must be massless and form $S U_{V}(5)$ color singlets. There is no interaction except gravitation among the $S U_{V}(5)$ color singlets so that they cannot form any object. The $S U_{V}(5)$ color singlets have only cosmological effects and cannot be found. It is seen that the $S U_{V}(5)$ color singlets are equivalent to the so-called dark energy. The $s$-particles and the $v$-particles can transform from one to another only when $T \sim T_{\max }$, where $T_{\max }$ is the highest temperature in the universeat which the symmetry is no breaking [9]. There is no singularity in the model; this model has solved the cosmological constant issue, and has explained the evolution of the universe and given some predicts.

According to the model, the gravitational field equation and the energy-momentum tensor are

$$
\begin{aligned}
& R_{\mu \nu}-\frac{1}{2} g_{\mu \nu} R=\kappa\left(T_{S \mu \nu}-T_{V \mu \nu}\right) \equiv \kappa T_{g \mu \nu}, \kappa=-8 \pi G, \\
& T_{\mu \nu}=T_{s \mu \nu}+T_{\nu \mu \nu}+T_{G \mu \nu}+T_{\Omega \mu \nu} .
\end{aligned}
$$

respectively, where $T_{g \mu v}, T_{s \mu \nu}, T_{v \mu \nu}, T_{G \mu v}$ and $T_{\Omega \mu v}$ are the tensor of the gravitational mass, $s$ - and $v$-energymomentum tensors, the energy-momentum tensor of the gravitational field and the energy-momentum tensor of the $\Omega$-Higgs field [9] respectively, $g_{\mu v}=\operatorname{diag}(-1,1,1,1)$ in flat space. According to [9], there is no contribution of $T_{G \mu \nu}$ and $T_{\Omega \mu \nu}$ to $G_{\mu \nu}$. If there is the contribution of $T_{G \mu v}$ to $G_{\mu \nu}, T_{g \mu v}$ should be correct.

Formula (2.3) in [9] corresponding to (1) has an obvious slip of a pen. Right formula (2.3) in [9] should be

$$
I_{S M}=\int \mathrm{d}^{4} x \sqrt{-g} \mathcal{L}_{S M}, \quad \mathcal{L}_{S M}=\alpha\left(\mathcal{L}_{s}+V_{0}\right)+\beta \mathcal{L}_{v}+\left(\frac{1}{2}\right) V_{s v} .
$$

In Section 2, the definition of the local energy-momentum tensor is given; Section 3 is the conclusion.

\section{The Definition of the Locally Conserved Energy-Momentum Tensor}

\subsection{The Difficulty to Solve the Issue of Local Conservation of Energy-Momentum in the General Relativity}

Let $t_{\mu v}$ be the energy-momentum tensor of the gravitational field, the field equation is

$$
R_{\mu \nu}-\frac{1}{2} g_{\mu \nu} R=\kappa\left(T_{\mu \nu}+t_{\mu \nu}\right),
$$

$\left(T^{\mu v}+t^{\mu v}\right)$ must simultaneously satisfy the following two equations,

$$
\begin{aligned}
& \left(T^{\mu \nu}+t^{\mu \nu}\right)_{; v}=0, \\
& \left(T^{\mu v}+t^{\mu \nu}\right)_{, v}=0 .
\end{aligned}
$$

It is not inevitable that one tensor $T^{\prime \mu v}=\left(T^{\mu v}+t^{\mu v}\right)$ satisfies simultaneously the two Equations (4) and (5). In fact [10],

$$
T_{\mu ; \nu}^{\prime v}=\frac{1}{\sqrt{-g}}\left(\sqrt{-g} T_{\mu}^{\prime \nu}\right)_{, \nu}-\Gamma_{\lambda, \nu \mu} T^{\prime v \lambda}
$$

(4) and (5) imply

$$
\Gamma_{\lambda, \nu \mu} T^{\prime \nu \lambda} \equiv 0
$$

But $\Gamma_{\lambda, \nu \mu}$ is not a tensor, hence (7) is not inevitable. This is a difficulty to define a locally conserved energymomentum. A possibility to evade the difficulty is that $t^{\mu v}$ is a quasi-local energy-momentum tensor. It can be seen from (7) that if $\left(T^{\mu v}+t^{\mu \nu}\right)$ is a quasi-local energy-momentum tensor, it possibly satisfies simultaneously 
the two Equations (4) and (5), because this implies a non-relativistic and independent quantity (according to the relativity, there is no action at a distance) to be added to $T^{\mu \nu}$ in this case.

\subsection{A Definition of the Energy-Momentum Tensor T $\mu v$ Which Is Locally Conserved in This Model without Singularity}

Analogously to the electromagnetic field, we define the energy-momentum tensor $T_{G \mu v}$ of the gravitational field to be

$$
T_{G \mu \nu}=\frac{1}{4 \pi S}\left(R^{\mu \alpha \beta \gamma} R_{\alpha \beta \gamma}^{v}-\frac{1}{4} g^{\mu \nu} R^{\lambda \alpha \beta \gamma} R_{\lambda \alpha \beta \gamma}\right),
$$

where $S$ is a constant with its dimension $[S]=\left[T G^{2}\right]$. It is obvious that $T_{G \mu \nu}$ is a tensor.

If there is the contribution of $T_{G \mu v}$ to $G_{\mu \nu}, T_{g \mu v}$ should be correct as

$$
T_{g \mu v}=T_{s \mu \nu}-T_{v \mu \nu}+\zeta T_{G \mu v},
$$

where $\zeta$ is a dimensionless number and is not determined for a time. $\zeta=0$ implies that there is no contribution of $T_{G \mu \nu}$ to $G_{\mu \nu} ; \zeta \neq 0$ implies that there is contribution of $T_{G \mu \nu}$ to $G_{\mu \nu}$.

The total energy-momentum tensor $T_{\mu v}$ is

$$
T_{\mu v}=T_{s \mu v}+T_{v \mu v}+T_{G \mu v}+T_{\Omega \mu v} .
$$

$T_{g \mu v}$ satisfies the gravitational field equation

$$
R_{\mu \nu}-\frac{1}{2} g_{\mu \nu} R=\kappa T_{g \mu v}=\kappa\left(T_{s \mu \nu}-T_{v \mu \nu}+\zeta T_{G \mu v}\right),
$$

thereby

$$
T_{g ; v}^{\mu \nu}=\left(R^{\mu v}-\frac{1}{2} g^{\mu \nu} R\right)_{; \nu}=0 .
$$

It is necessary that the energy-momentum tensor is locally conserved. Hence $T_{\mu v}$ should satisfy the equation

$$
\left(\sqrt{-g} T^{\mu \nu}\right)_{, v}=\left(\left[\sqrt{-g}\left(T_{s}^{\mu v}+T_{v}^{\mu v}+T_{G}^{\mu v}+T_{\Omega}^{\mu \nu}\right)\right], v\right)=0 .
$$

$T_{g v}^{\mu \nu}$ and $T_{\mu \nu}$ are two independent tensors, hence they can satisfy the Equations (13) and (14), respectively.

It is possible that the definition of $T_{G \mu v}$ is not best. But no matter which definition of $T_{G \mu v}$, provided it is a tensor, (13) and (14) can still hold. It is seen that the conservative law of energy-momentum can still hold in the general relativity.

\section{Conclusion}

According to the conventional theory it is difficult to define the energy-momentum tensor which is locally conventional. The energy-momentum tensor of the gravitational field is defined. Based on [9], the total energymomentum tensor $T^{\mu v}$ is defined which satisfies $\left(\sqrt{-g} T^{\mu v}\right)_{, v}=0$. Consequently, the locally conservative law of energy-momentum can still hold in the general relativity. The energy-momentum tensor $T_{g}^{\mu \nu}$ of the gravitational mass is different from $T^{\mu \nu}$. $T_{g}^{\mu \nu}$ satisfies the gravitational field equation and its covariant derivative is zero.

\section{Acknowledgements}

This work is supported by the National Natural Science Foundation of China (Grant Nos. 11175032, 61475033). I am very grateful to Professor Zhao Zhanyue and Professor Wu Zhaoyan for their helpful discussions and best support.

\section{References}

[1] Einstein, A. (1915) Berl Ber, 178; (1918) Berl Ber, 448. 
[2] Tolman, R.C. (1930) Physical Review, 35, 875.

[3] Bauer, H. (1918) Physik Z, 19, 163. http://dx.doi.org/10.1103/PhysRev.35.875

[4] Landau, L.D. and Lifshitz, E.M. (1999) The Classical Theory of Fields. 4th Edition, Butterworth-Heinemann, Beijing, 280.

[5] Mpller, C. (1961) Annals of Physics, 12, 118-133.

[6] Brown, J.D. and York Jr., J.W. (1993) Physical Review D, 47, 1407. http://dx.doi.org/10.1103/PhysRevD.47.1407

[7] Chen, C.M. and Nester, J.M. (1993) Classical and Quantum Gravity, 16, 1279. http://dx.doi.org/10.1088/0264-9381/16/4/018

[8] Hecht, R.D. and Nester, J.M. (1993) Physics Letters A, 180, 324. http://dx.doi.org/10.1016/0375-9601(93)91185-8

[9] Chen, S.-H. (2014) International Journal of Astronomy and Astrophysics, 4, 264-293. http://dx.doi.org/10.4236/ijaa.2014.41023

[10] Liu, L. and Zhao, Z. (2004) General Relativity (in Chinese). Higher Education Press, Beijing, 110. 